\title{
Length Scales of Eddy Generation and Nonlinear Evolution of the Seasonally Modulated South Pacific Subtropical Countercurrent
}

\author{
Bo QIU \\ Department of Oceanography, University of Hawaii at Manoa, Honolulu, Hawaii \\ Robert B. SCOTT \\ Institute for Geophysics, The University of Texas at Austin, Austin, Texas \\ SHUIMING CHEN \\ Department of Oceanography, University of Hawaii at Manoa, Honolulu, Hawaii
}

(Manuscript received 12 June 2007, in final form 26 November 2007)

\begin{abstract}
The dynamical processes behind the seasonal modulation of the two-dimensional eddy kinetic energy (EKE) wavenumber spectrum in the Subtropical Countercurrent region of the South Pacific are investigated with $14 \mathrm{yr}$ of satellite altimeter data and climatological hydrographic data. The authors find a seasonally modulated generation of EKE via baroclinic instability in modes with larger meridional length scales. Subsequent nonlinear eddy-eddy interactions redistribute the EKE to larger total horizontal length scales, and larger zonal scales in particular. This is confirmed by diagnosing the spectral transfer of EKE in the surface geostrophic flow, which is found to drive an anisotropic inverse cascade, being redirected in the sense consistent with the $\beta$ effect, as predicted by geostrophic turbulence theory on the $\beta$ plane. Because of the seasonal renewal of meridionally elongated anomalies by baroclinic instability and possibly because of the barotropization process, however, the net outcome for the formation of surface zonal flows is observed to be limited.
\end{abstract}

\section{Introduction}

Accumulation of high-quality sea surface height measurements from satellite altimeters over the past oneand-a-half decades has provided us not only a tool to monitor the ocean circulation variability on the global scale, but also a means to explore the dynamics underlying the detected variability. It is well known that the oceanic variability is dominated by mesoscale eddy signals with temporal and spatial scales at approximately 50-200 days and 100-500 km, respectively (e.g., Robinson 1983). Many recent studies have indeed taken advantage of the concurrent satellite altimeter missions to examine various aspects of the mesoscale eddy signals. A comprehensive review of these studies was con-

Corresponding author address: Dr. Bo Qiu, Department of Oceanography, University of Hawaii at Manoa, 1000 Pope Road, Honolulu, HI 96822.

E-mail: bo@soest.hawaii.edu ducted by Le Traon and Morrow (2001) and recently updated by Morrow and Le Traon (2006).

Despite a steady increase in our knowledge of the mesoscale eddy signals, one area where our understanding remains fragmentary is how mesoscale eddies interact among themselves and to what extent this interaction contributes to the flow of various length scales. An example of this limited understanding is well illustrated in the Subtropical Countercurrent (STCC) bands of the South and North Pacific Oceans. In these bands of the wind-driven subtropical gyres, elevated eddy kinetic energy (EKE) level has been observed to modulate seasonally with an EKE peak appearing in the local spring season: April-May in the North Pacific STCC band and November-December in the South Pacific STCC band (see Fig. 1, with more details in section 2). This spring-season, increased EKE is the result of the enhanced baroclinic instability between the vertically sheared, eastward-flowing STCC and the subsurface, westward-flowing North (or South) Equatorial 

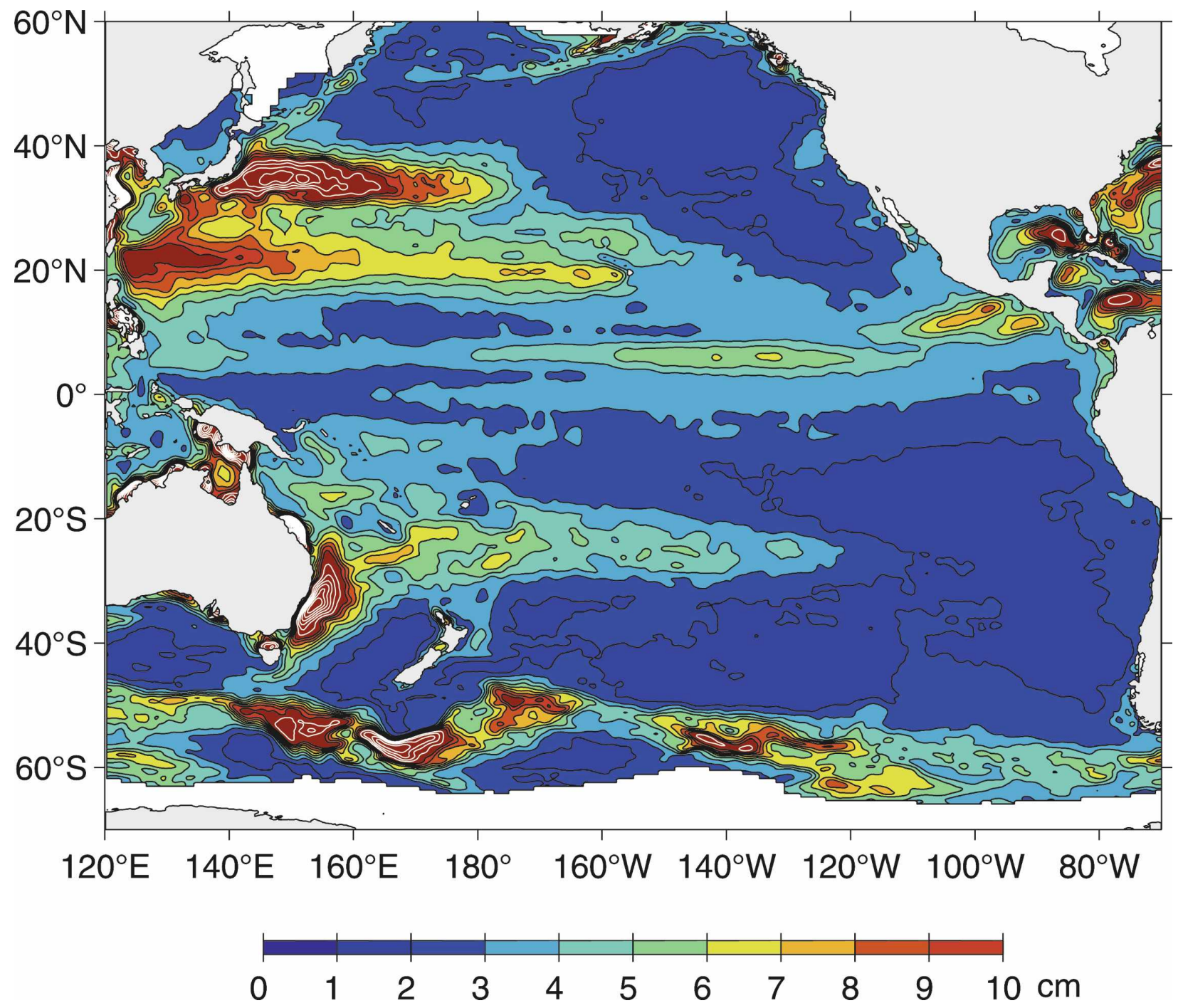

FIG. 1. RMS sea surface height variability in the Pacific Ocean, based on high-pass-filtered satellite altimeter SSH data from October 1992 to December 2006. The high-pass filter has a half-power at 180 days. Contour intervals are $0.01 \mathrm{~m}$ for black lines, and $0.02 \mathrm{~m}$ for white lines starting from $0.12 \mathrm{~m}$. The STCC band in the South Pacific corresponds to the high RMS SSH region of $20^{\circ}-30^{\circ} \mathrm{S}$, $167^{\circ} \mathrm{E}-130^{\circ} \mathrm{W}$.

Current (e.g., Qiu 1999; Roemmich and Gilson 2001; Kobashi and Kawamura 2002; Qiu and Chen 2004; Bowen et al. 2005). However, the role of eddy interaction in the subsequent nonlinear evolution is unclear. In particular, how is the surface EKE dissipated? Is EKE transferred down the water column where large-scale flow can be dissipated via interaction with the bottom topography? Or, does surface EKE cascade to small scales, implying that most EKE is likely dissipated in the upper depths where the EKE levels are high? These questions have implications for the various features of the ocean circulation ranging from what sets the length scales of the surface flow to where diapycnal mixing occurs.
To a great extent, clarifying the interaction and decay processes of the mesoscale eddies is of equal importance to knowing their generation mechanisms. The processes, after all, determine the equilibrium "turbulent" state of the world's oceans. The objective of our present study is to explore the eddy interaction and decay processes by focusing on the STCCs in the Pacific Ocean. We hope to make progress on these challenges by studying the anisotropy and length scales of eddy generation and subsequent evolution on seasonal time scales. These techniques are commonly employed in idealized dynamical studies, but are rarely applied to observational oceanographic data analysis. There are two reasons to choose the STCCs as our starting explo- 
ration site. Other high-EKE regions in the Pacific Ocean, for example, include the western boundary currents (the Kuroshio and its extension in the North Pacific, and the East Australian Current in the South Pacific) and the Antarctic Circumpolar Current in the Southern Ocean (Fig. 1). In both of these regions, eddy interaction processes are likely to be more complicated because of the barotropic nature of the strong currents and their interactions with the neighboring recirculation gyre and bottom topography. The second reason for focusing on the STCCs is that their clear annual cycle in the EKE field presents us with a unique opportunity to separate the generation, interaction, and decay processes in the observed eddy signals.

A great deal about generic eddy interaction and nonlinear flow evolution has been learned from idealized geostrophic turbulence models, for both freely decaying, horizontally homogeneous turbulence (e.g., Rhines 1977; Vallis and Maltrud 1993; Smith and Vallis 2001) and the statistical steady state of homogeneous turbulence forced by a vertically sheared eastward mean flow (e.g., Panetta 1993; Smith and Vallis 2002; Arbic and Flierl 2004). A rich phenomenology has developed (Danilov and Gurarie 2000; Vallis 2006), with key features being a robust inverse cascade transferring total (kinetic plus potential) energy toward the deformation scale and toward lower baroclinic modes. The energy, after finally reaching the barotropic mode, is ultimately transferred toward larger scales via the classic barotropic inverse kinetic energy cascade (Charney 1971; Fu and Flierl 1980; Salmon 1980; Hua and Haidvogel 1986; Larichev and Held 1995; Salmon 1998; Smith and Vallis 2001). On a spherical planet the (meridional) planetary potential vorticity gradient $\beta$ may dominate, which tends to redirect the barotropic inverse cascade more into larger-scale zonally oriented flow at the expense of meridionally oriented flow (Rhines 1977; Vallis and Maltrud 1993; Galperin et al. 2004; 2006). It is important to emphasize that the classic inverse cascade, because it is for the barotropic mode only, will likely not be apparent in the altimeter data because the surface dynamics largely reflects the first-baroclinic-mode motions (Wunsch 1997).

Recently Scott and Arbic (2007, hereafter SA07) found that, analogous to the barotropic inverse cascade, there can also be an inverse cascade of baroclinic kinetic energy. This does not contradict the well-known forward cascade of total baroclinic energy above the deformation scale (Charney 1971; Salmon 1998; also S. Danilov 2006, personal communication), because it turns out the forward potential energy cascade slightly overpowers the inverse kinetic energy (KE) cascade, leaving a small residual cascade of total energy con- verging toward the deformation scale. Because this baroclinic KE cascade dominates the surface layer cascade of the two-layer idealized quasigeostrophic (QG) model with surface-intensified stratification (thinner upper layer) used by SA07, it likely reflects the KE cascade observable with the altimeter data at least at scales larger than the deformation radius; at smaller scales, surface geostrophic turbulence effects become important (Lapeyre and Klein 2006). The inverse cascade of baroclinic KE was forced by baroclinic instability and "arrested" by barotropization (i.e., the transfer of energy to the lower layer to form more depth-independent motion). SA07 was based on homogeneous $f$-plane simulations, so it was not possible to study the $\beta$ effect on cascade direction. It remains an open question whether or not this baroclinic inverse kinetic energy cascade is redirected by $\beta$, analogous to the $\beta$ effect on the baroclinic inverse cascade. In other words, it is not clear whether or not this inverse cascade should drive zonally oriented jets at the surface of the ocean. It is worth noting that the reverse is also true; if we observe a zonally oriented jet at the surface, it is not clear whether an inverse cascade is driving it, since $\beta$ can introduce an anisotropy even without a cascade.

By averaging the altimetrically derived surface zonal velocity over an 18-week window, Maximenko et al. (2005) observed zonally coherent jetlike structures in the World Ocean. Interestingly, the clearest areas where the time-averaged jet structures show up are in the STCC bands. Important questions remain: Are these jets formed through dynamic processes described in the previous paragraph? If not, how do they arise?

One effective diagnostic tool to assess the nonlinear eddy interaction is the scale-by-scale energy budget analysis advocated by Frisch (1995, chapter 2). This method was applied by Scott and Wang (2005, hereafter SW05) to reveal an inverse kinetic energy cascade in the South Pacific Ocean from satellite altimetric measurements. In this study, we extend SW05 by looking into the detailed seasonal evolution of the mesoscale eddies in the STCC band of the South Pacific Ocean. We provide concrete evidence supporting baroclinic instability as the mechanism driving the inverse energy cascade diagnosed by SW05. By exploring the seasonal evolution of the energy budget at various length scales, we attempt to clarify the dynamic processes governing the mesoscale eddy generation and nonlinear evolution in the STCC regions.

\section{EKE signals in the STCC bands}

For this study we use the global sea surface height (SSH) dataset compiled by the Collecte Localisation 


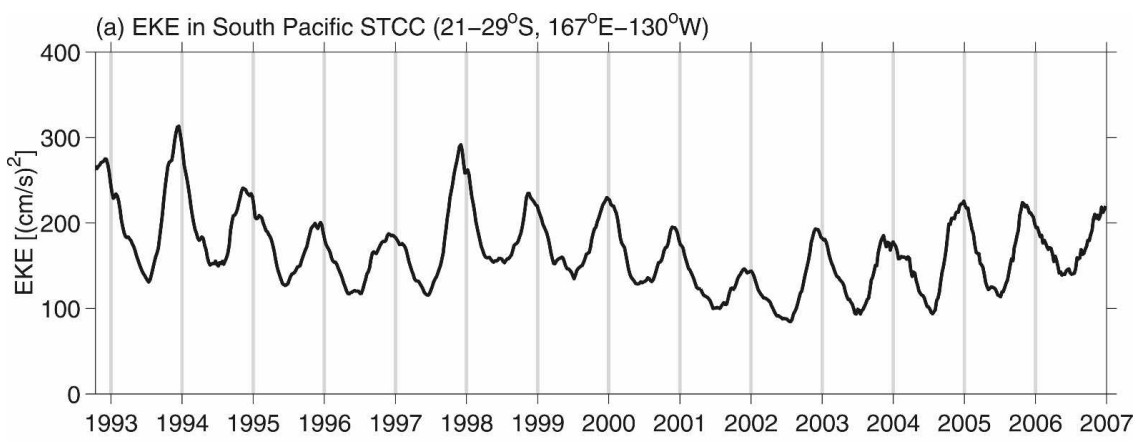

(b) EKE in North Pacific STCC $\left(18-28^{\circ} \mathrm{N}, 135^{\circ} \mathrm{E}-175^{\circ} \mathrm{W}\right)$

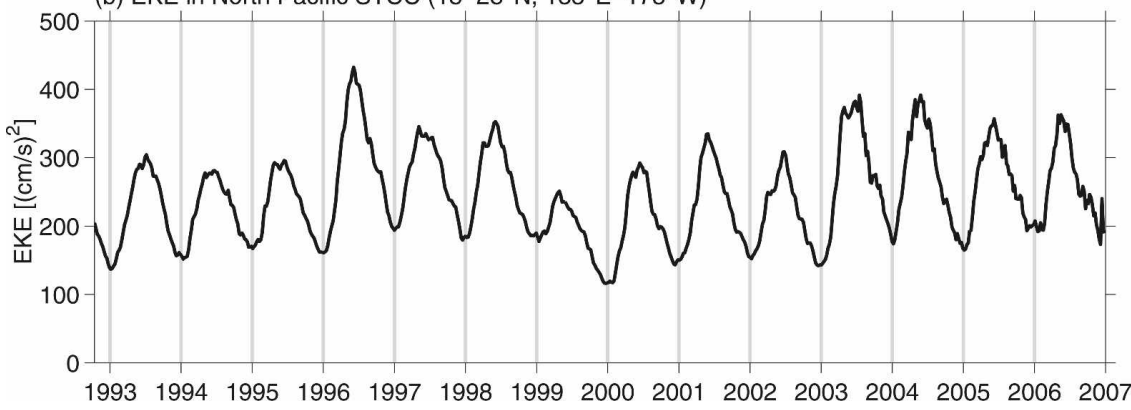

FIG. 2. Eddy kinetic energy time series in the regions of (a) South Pacific Subtropical Countercurrent $\left(21^{\circ}-29^{\circ} \mathrm{S}, 167^{\circ} \mathrm{E}-130^{\circ} \mathrm{W}\right)$ and (b) North Pacific Subtropical Countercurrent $\left(18^{\circ}-28^{\circ} \mathrm{N}, 135^{\circ} \mathrm{E}-175^{\circ} \mathrm{W}\right)$.

Satellites (CLS) Space Oceanography Division of Toulouse, France. The dataset merges all available satellite altimeter along-track SSH measurements for the period from October 1992 through December 2006. It has a 7 -day temporal resolution and a $1 / 3^{\circ}$ longitude Mercator grid resolution. For the detailed mapping method and data quality analysis, see Le Traon et al. (1998) and Ducet et al. (2000).

Figure 2a shows the EKE time series averaged in the South Pacific STCC band of $21^{\circ}-29^{\circ} \mathrm{S}, 167^{\circ} \mathrm{E}-130^{\circ} \mathrm{W}$. Here, EKE is calculated from the gridded SSH anomaly data $h^{\prime}$ by assuming geostrophy:

$$
\mathrm{EKE} \equiv \frac{g^{2}}{2 f^{2}}\left[\left(\frac{\partial h^{\prime}}{\partial x}\right)^{2}+\left(\frac{\partial h^{\prime}}{\partial y}\right)^{2}\right]
$$

where $g$ is the gravity constant and $f$ the Coriolis parameter. While interannual variability also exists, the EKE level in Fig. 2a is clearly dominated by a seasonal modulation with a maximum in November-December and a minimum in June-July. Compared to the mean EKE level, $172 \mathrm{~cm}^{2} \mathrm{~s}^{-2}$, in this band, the seasonal EKE modulation has a peak-to-peak amplitude of $\sim 100$ $\mathrm{cm}^{2} \mathrm{~s}^{-2}$. Note that a very similar seasonal EKE modulation is also detected in the North Pacific STCC band of $18^{\circ}-28^{\circ} \mathrm{N}, 135^{\circ} \mathrm{E}-175^{\circ} \mathrm{W}$ (Fig. $2 \mathrm{~b}$ ). Like its counter- part in the Southern Hemisphere, the maximum EKE is observed during the local spring season in April-May. ${ }^{1}$

It is worth mentioning that the South Pacific STCC band is dominated by westward-propagating mesoscale eddies (see, e.g., Fig. 14 in Qiu and Chen 2004). The weakening in the EKE level shown in Fig. 2a, however, is not due to the mesoscale eddies generated in September-December leaving the region of our interest. A look at the meridionally averaged EKE distribution from $20^{\circ}$ to $30^{\circ} \mathrm{S}$ as a function of time and longitude (Fig. 3) reveals that the eddies generated in September-December "disappear" mostly locally.

To further clarify the seasonal EKE evolution in the South Pacific STCC band, we plot in Fig. 4 the bimonthly EKE power spectral density, $\bar{E}\left(k_{x}, k_{y}, t\right)$, as a function of zonal and meridional wavenumber, $k_{x}$ and $k_{y}$. For these calculations, the SSH anomaly data were preprocessed as follows (for the spectral transfers described below, the absolute SSH data were preprocessed identically): The SSH anomaly data were partitioned into 12 overlapping boxes with $32 \times 32$ grid points, centered at $25^{\circ} \mathrm{S}$, and every $5^{\circ}$ longitude from

\footnotetext{
${ }^{1}$ Most of the results presented in this study for the South Pacific STCC are valid for the North Pacific STCC. For brevity, we restrict our presentations to those for the South Pacific STCC.
} 


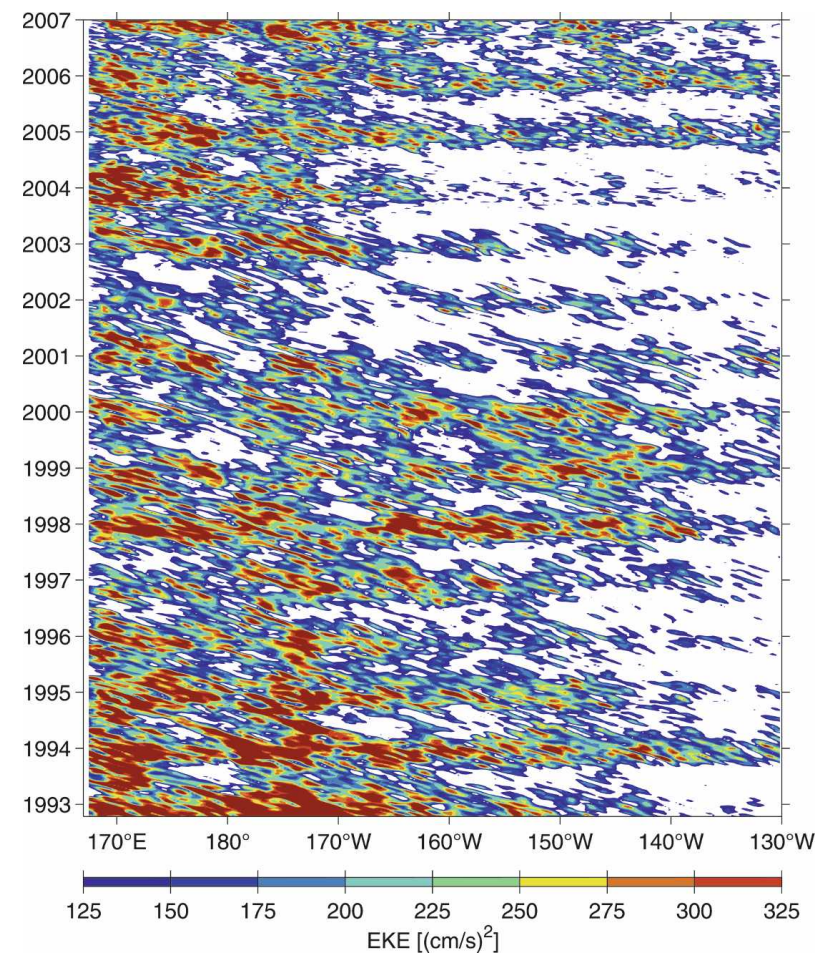

FIG. 3. Time-longitude plot of the eddy kinetic energy averaged in the zonal band of $21^{\circ}-29^{\circ} \mathrm{S}$. White areas indicate the EKE level $<125 \mathrm{~cm}^{2} \mathrm{~s}^{-2}$. $170^{\circ} \mathrm{E}$ to $135^{\circ} \mathrm{W}$. For each box and each week, the SSH anomaly fields were first detrended by fitting a linear plane via least squares, and subtracting this plane from the corresponding week. A Hanning window was applied to the detrended data. The averages were over all available weeks and all 12 boxes within the STCC band.

During the initial eddy growth phase of October-November (Fig. 4a), it appears that the eddy signals have a slightly higher energy level at large $k_{x}$ than at large $k_{y}$, implying that the mesoscale eddies at this phase are more meridionally, than zonally, elongated. This was confirmed by integrating $\bar{E}\left(k_{x}, k_{y}, t\right)$ over the two regions $k_{y}>k_{x}$ and $k_{x}>k_{y}$ to form

$$
\begin{aligned}
\mathrm{EKE}_{z} & =\sum_{k_{y}>k_{x}} \bar{E}\left(k_{x}, k_{y}, t\right) \Delta k^{2}, \\
\mathrm{EKE}_{m} & =\sum_{k_{x}>k_{y}} \bar{E}\left(k_{x}, k_{y}, t\right) \Delta k^{2},
\end{aligned}
$$

where $\bar{E}\left(k_{x}, k_{y}, t\right)$ is the bimonthly averages shown in Fig. 4. Figure 5 shows $\left(\mathrm{EKE}_{z}-\mathrm{EKE}_{\mathrm{m}}\right) / \mathrm{EKE}$; for clarity, the annual cycles are repeated twice. Note that October-November is the only time for which the flow is more meridionally aligned. During November-December when the EKE level reaches the seasonal maximum, the situation starts to reverse in Fig. 4: more eddy variance appears at larger $k_{y}$ than $k_{x}$. This is reflected in
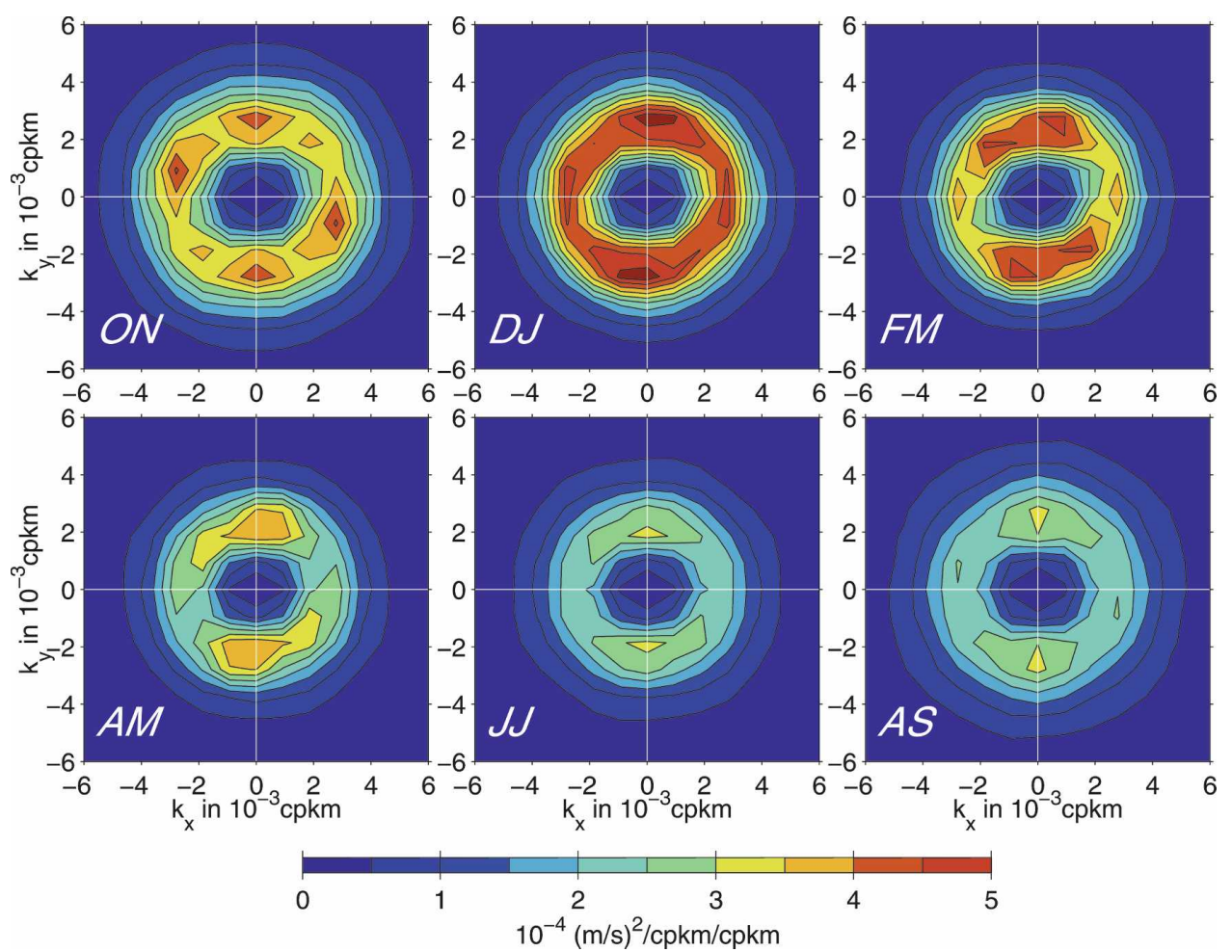

FIG. 4. Bimonthly EKE power spectral density distributions as a function of $k_{x}$ and $k_{y}$ in the South Pacific STCC band of $20^{\circ}-30^{\circ} \mathrm{S}, 170^{\circ} \mathrm{E}-130^{\circ} \mathrm{W}$. The two-letter monthly designators start with OctoberNovember (ON) in the top left and conclude with August-September (AS) in the lower right. 


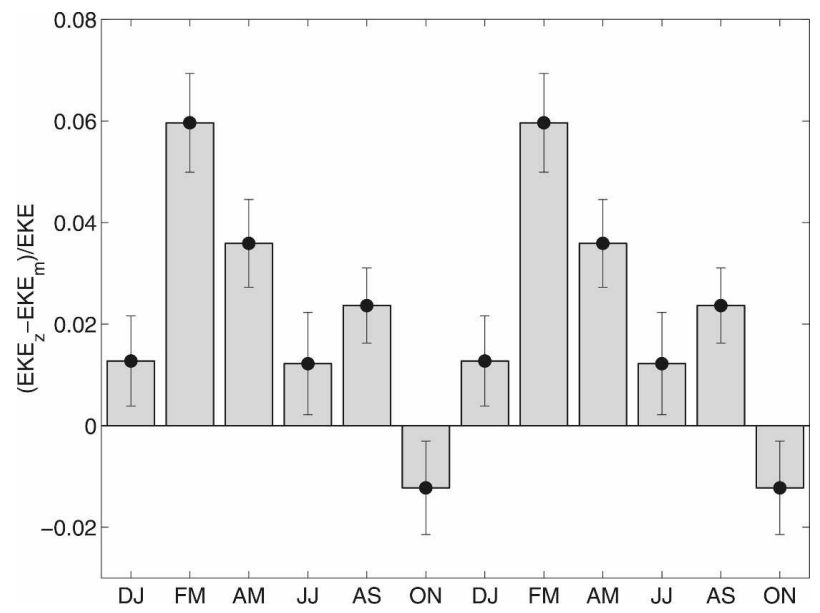

FIG. 5. Measure of proportion of EKE that is zonally elongated vs meridionally elongated $\left(\mathrm{EKE}_{z}-\mathrm{EKE}_{m}\right) / \mathrm{EKE}$. See Eqs. (2) and (3) for definitions. Error bars represent the std dev divided by $\sqrt{N-1}$, where $N$ is the number of available EKE estimates in the bimonthly bins.

Fig. 5 with $\mathrm{EKE}_{z}>\mathrm{EKE}_{m}$. The preference for more EKE at larger zonal scales than meridional scales continues in the subsequent months from February to September. During the same period, there is a persistent drop in the overall energy level of the mesoscale eddies and it is not until September that the EKE level starts to increase, and again with a preference for large $k_{x}$. In the following two sections we interpret this evolution of $\bar{E}\left(k_{x}, k_{y}, t\right)$ in terms of generation by baroclinic instability and an anisotropic EKE cascade via nonlinear triad interactions, respectively.

A visual comparison between the power spectral density $\bar{E}\left(k_{x}, k_{y}, t\right)$ of June-July and OctoberNovember along the $k_{y}$ axis in Fig. 4 reveals that the EKE peak has a smaller $k_{y}$ value in the June-July eddy decay period $\left(k_{y} \sim 2.0 \times 10^{-3} \mathrm{cpkm}\right)$ than in the October-November eddy growth period $\left(k_{y} \sim 2.8 \times 10^{-3}\right.$ $\mathrm{cpkm} ; \mathrm{cpkm}$ is cycles per kilometer). To quantitatively examine the seasonal variations in the energy-containing length scale, we define

$$
L_{e}(t)=2 \pi \sum_{k_{x}, k_{y}} \bar{E}\left(k_{x}, k_{y}, t\right) \Delta k^{2} / \sum_{k_{x}, k_{y}} K \bar{E}\left(k_{x}, k_{y}, t\right) \Delta k^{2},
$$

where $K=\sqrt{k_{x}^{2}+k_{y}^{2}}$ is the total wavenumber, and plot its monthly time series in Fig. 6. The time series shows $L_{e}$ has a maximum in March-April and a minimum in September. Notice that this annual cycle for $L_{e}$ lags that for EKE (see Fig. 2a or the dashed line in Fig. 8) by $\sim 3$ months.

Using a different definition for $L_{e}$, Kobashi and Kawamura (2002) examined the energy-containing eddy length scales in the North Pacific STCC band. A

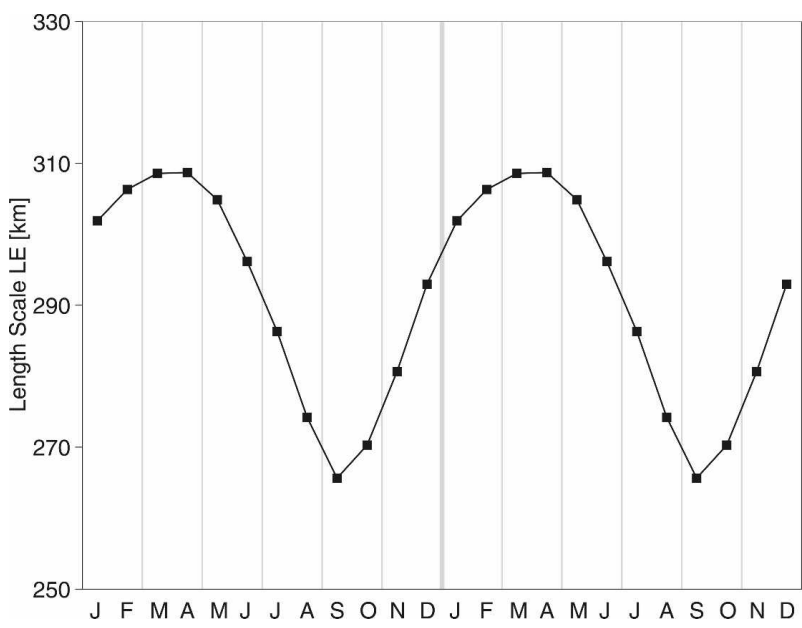

FIG. 6. Energy-containing length scale, $L_{e}$, estimated from Eq. (4) vs month. Here, the length scale is in terms of wavelengths.

similar 2-3-month lag in $L_{e}$ behind the regional EKE annual cycle was detected (see their Fig. 10a). This delayed increase in eddy length scales following the EKE peak was suggested by Kobashi and Kawamura (2002) to reflect the eddy-eddy interaction, although no analysis was provided to verify this suggestion.

\section{Eddy growth and baroclinic instability}

The seasonally varying EKE level detected in the South Pacific STCC band has been identified by Qiu and Chen (2004, hereafter QC04) as reflecting the regional baroclinic instability whose intensity modulates on the seasonal time scale. In this section, we briefly review QC04 and provide an update on the results that are relevant to our eddy interaction analysis pursued in section 4.

Figure 7 shows the thermal structures across the western subtropical gyre of the South Pacific in the four months representing the different seasons. In the $20^{\circ}-$ $30^{\circ} \mathrm{S}$ band of our interest, the isotherms below the 250-m depth have in general a downward slope toward the south; these sloping isotherms represent the westward-flowing South Equatorial Current in the winddriven South Pacific subtropical gyre (SEC; Roemmich and Cornuelle 1990; Huang and Qiu 1998). In the surface-250-m layer, the isotherms tilt upward toward the south as a result of Ekman heat transport convergence and latitudinally dependent surface buoyancy forcing. These upward-tilting isotherms give rise to the eastward-flowing STCC (e.g., Rotschi 1973). Subject to the seasonal surface wind/buoyancy forcing, the tilt of the upper-ocean isotherms (or the intensity of STCC) undergoes a clear annual cycle: the tilt is largest in late 

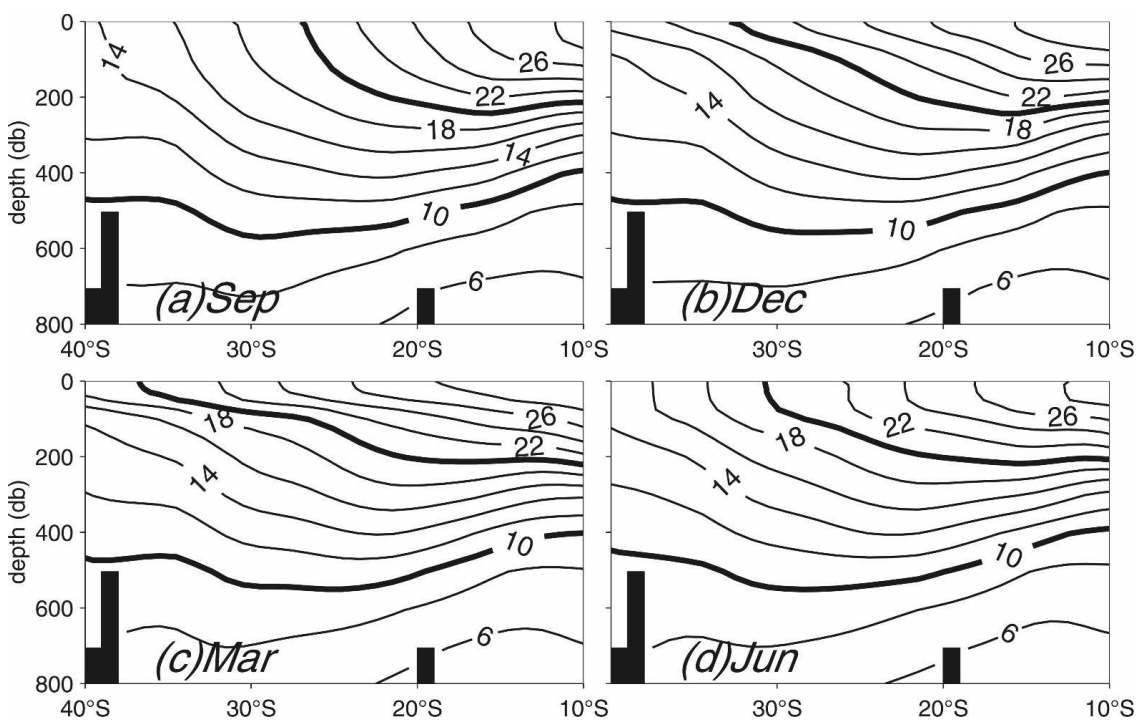

FIG. 7. Seasonal evolution of the thermal structures associated with the STCC-SEC along $170^{\circ} \mathrm{E}$, based on the monthly climatological temperature-salinity dataset of the World Ocean Atlas 2005 (Locarnini et al. 2006; Antonov et al. 2006). The contour unit is ${ }^{\circ} \mathrm{C}$.

austral winter (Fig. 7a) following the accumulative wind/buoyancy forcing, and it flattens out in austral summer (Fig. 7c) after the surface buoyancy forcing changes from cooling to heating.

The consequence of this seasonal progression in the upper-ocean thermal structures is that the velocity shear between the STCC and SEC exhibits a regular annual cycle. The solid line in Fig. 8 shows the velocity shear between the surface STCC and the SEC at the

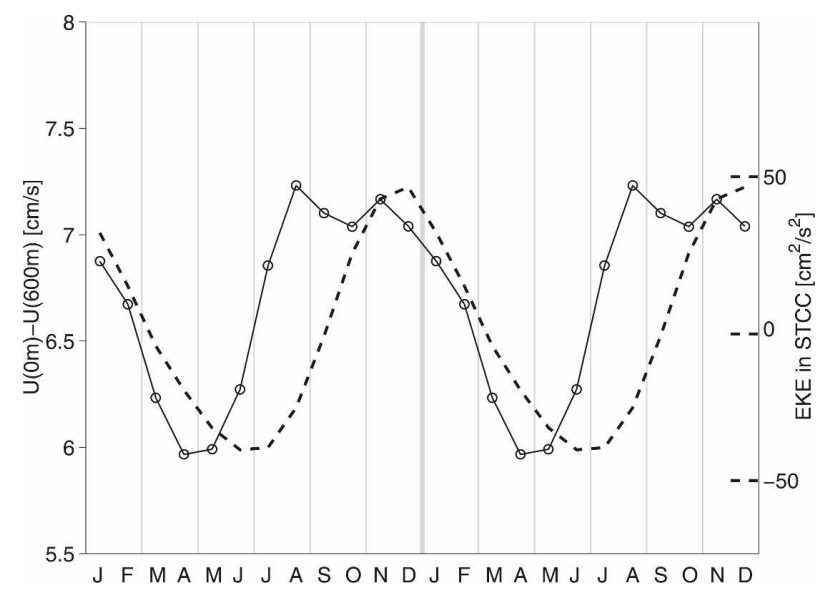

FIG. 8. Zonal velocity shear between 0 and $600 \mathrm{~m}$ averaged over the STCC-SEC region of $21^{\circ}-29^{\circ} \mathrm{S}, 180^{\circ}-160^{\circ} \mathrm{W}$ (solid line) vs month. The zonal flows are calculated from the monthly mean temperature-salinity dataset of the World Ocean Atlas 2005 (Locarnini et al. 2006; Antonov et al. 2006) with a three-point smoothing $(1 / 4,1 / 2,1 / 4)$ applied. The dashed line shows the monthly EKE climatology in the STCC region based on Fig. 2a. 600-m depth. The velocity shear is largest in August when the surface-cooling-induced isotherm tilt reaches the maximum. The weakest shear is found in AprilMay when the surface ocean is capped by a flat and well-stratified seasonal thermocline. As can be seen in Fig. 8, the annual cycle of the STCC-SEC velocity shear leads the EKE annual cycle (the dashed line in Fig. 8) by about 3-4 months.

By adopting a 2.5-layer reduced-gravity model, QC04 indicated that the observed EKE annual cycle can be understood as the outcome of baroclinic instability in the vertically sheared STCC-SEC system. Specifically, the linearized equations governing the perturbation potential vorticity $q_{n}(n=1,2)$ in the 2.5 -layer reducedgravity model are

$$
\left(\frac{\partial}{\partial t}+U_{n} \frac{\partial}{\partial x}\right) q_{n}+\frac{\partial \Pi_{n}}{\partial y} \frac{\partial \phi_{n}}{\partial x}=0,
$$

where $\phi_{n}$ is the perturbation streamfunction, $U_{1}\left(U_{2}\right)$ is the zonal mean velocity for STCC (SEC), and $\Pi_{n}$ is the mean potential vorticity (e.g., Pedlosky 1987). Assuming $U_{n}=$ const, $q_{n}$ and the meridional gradient of $\Pi_{n}$ are given by

$$
\begin{aligned}
q_{n} & =\nabla^{2} \phi_{n}+\frac{(-1)^{n}}{\gamma_{2} \delta_{n} \lambda^{2}}\left(\phi_{1}-\phi_{2}-\gamma_{n} \phi_{2}\right), \\
\Pi_{n y} & =\beta-\frac{(-1)^{n}}{\gamma_{2} \delta_{n} \lambda^{2}}\left(U_{1}-U_{2}-\gamma_{n} U_{2}\right),
\end{aligned}
$$

where $\delta_{n}=H_{n} / H_{2}, \gamma_{n}=\left(\rho_{n}-\rho_{1}\right) /\left(\rho_{3}-\rho_{2}\right)$, and $\lambda^{2}=$ $g H_{2}\left(\rho_{3}-\rho_{2}\right) / \rho_{0} f_{0}^{2}$. In the above equations, $H_{n}$ denotes 
the mean $n$th layer thickness, $\rho_{n}$ the $n$th layer density, $\beta$ the meridional gradient of the Coriolis parameter, $f_{0}$ the Coriolis parameter at the reference latitude, and $\rho_{0}$ the reference density.

The stability of this 2.5-layer STCC-SEC system can be analyzed by seeking the normal-mode solution: $\phi_{n}=$ $\Re\left[A_{n} \operatorname{expi}\left(k_{x} x+k_{y} y-k_{x} c t\right)\right]$. Substituting $\phi_{n}$ into Eq. (5) and requiring nontrivial solutions for $A_{n}$ leads to a quadratic dispersion relation for the complex phase speed $c=c_{r}+i c_{i}$ [see Eq. (6) in QC04]. Two parameters in the surface STCC layer change significantly with seasons: $U_{1}$ related to the changing tilt of the surface layer isotherms, and $\rho_{1}$ related to the seasonally varying buoyancy forcing. In August-September, for example, $U_{1}=0.055 \mathrm{~m} \mathrm{~s}^{-1}$ and $\rho_{1}=24.8 \sigma_{\theta}$; in contrast, they are $0.04 \mathrm{~m} \mathrm{~s}^{-1}$ and $24.4 \sigma_{\theta}$ in April-May. Using these and other common parameter values appropriate for the STCC-SEC system (listed in the caption of Fig. 9), we compare in Fig. 9 the growth rates of the vertically sheared STCC-SEC system in these two opposite seasons. In both cases, long- and short-wave cutoffs exist for the unstable perturbations. The unstable window is much wider under the August-September condition where perturbations with wavelengths between 200 and $370 \mathrm{~km}$ are baroclinically unstable than under the April-May condition where the unstable wavelengths are limited to between 260 and $320 \mathrm{~km}$. Notice that the most unstable wave in August-September has a wavelength of $\sim 250 \mathrm{~km}$; this value is slightly smaller than the energy-containing wavelength value, $\sim 270 \mathrm{~km}$, estimated from the satellite altimeter data (recall Fig. 6). As will be quantified in section 4 , this increase in length scale is a result of inverse energy cascade through nonlinear eddy interactions.

Because of the greater shear between STCC and SEC and the weaker stratification, the August-September condition allows for a more vigorous eddy growth: the maximum growth rate has $e$-folding time scale, $\left(k_{x} c_{i}\right)^{-1} \sim 50$ days in Fig. 9a. In comparison, the maximum growth rate has $e$-folding time scale $>200$ days in April-May. It is worth noting that within the unstable window, perturbations with large $k_{x}$ and small $k_{y}$ tend to grow faster. In other words, during the growing phase of baroclinic instability, meridionally elongated anomalies would dominate the zonally elongated anomalies. The fact that the observed EKE is more meridionally, than zonally, aligned in October-November (recall Figs. 4 and 5) is consistent with this dynamic prediction.

\section{Diagnosis of eddy-eddy interaction}

Lengthening in the energy-containing scale $L_{e}$ following the growing stage of baroclinic instability (Fig. 6)
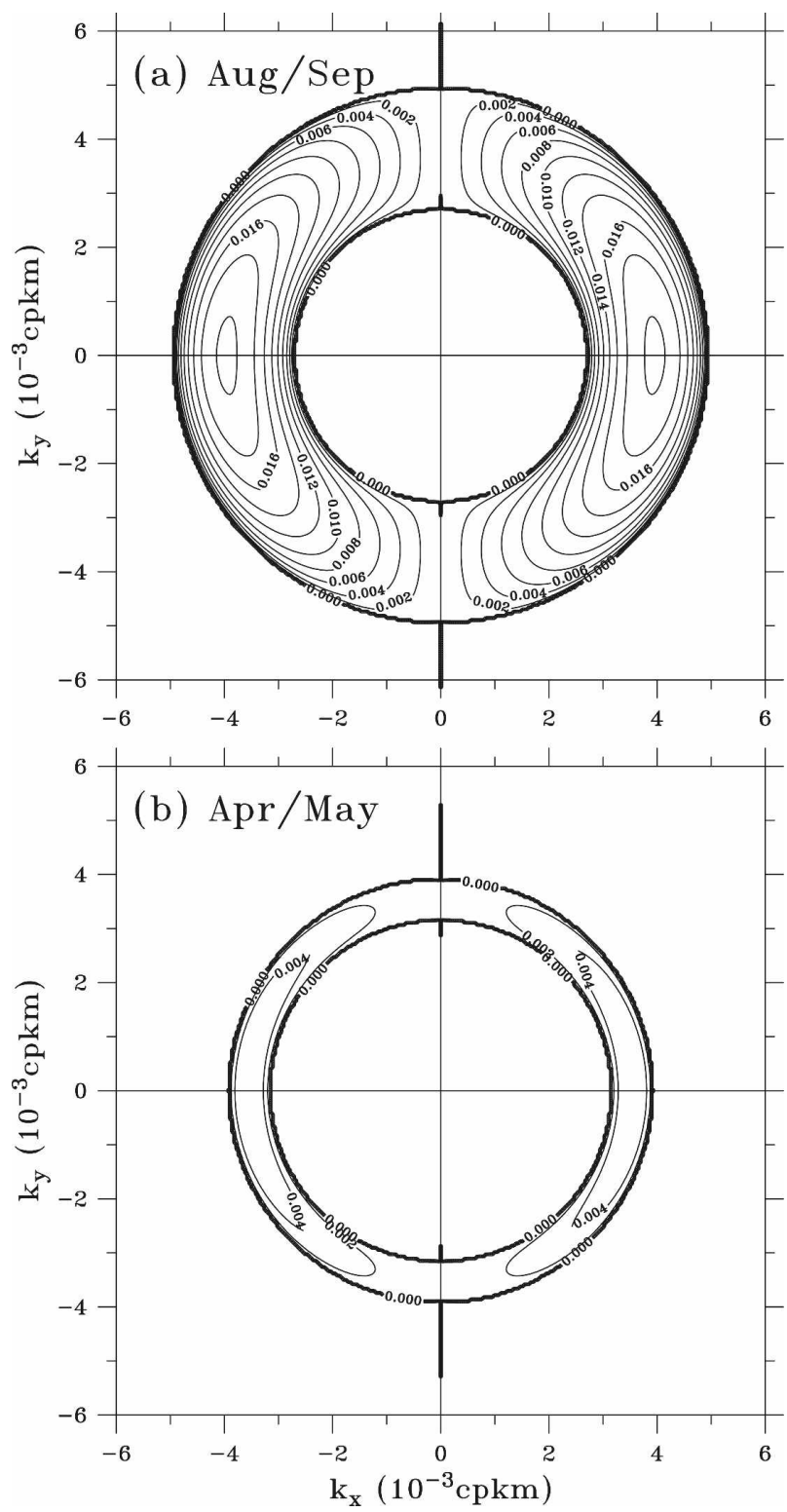

FIG. 9. Growth rate $\left(\mathrm{day}^{-1}\right)$ as a function of $k_{x}$ and $k_{y}$ for the STCC-SEC system in (a) August-September and (b) April-May. Regions outside of the concentric circles are baroclinically stable. Except for the $U_{1}$ and $\rho_{1}$ values (see text), other parameters have the same values in (a) and (b): $f_{0}=-6.15 \times 10^{-5} \mathrm{~s}^{-1}, \beta=2.08 \times$ $10^{-11} \mathrm{~s}^{-1} \mathrm{~m}^{-1}, U_{2}=-0.02 \mathrm{~m} \mathrm{~s}^{-1}, H_{1}=200 \mathrm{~m}, H_{2}=700 \mathrm{~m}, \rho_{2}=$ $26.60 \sigma_{\theta}$, and $\rho_{3}=27.75 \sigma_{\theta}$.

points to the possibility of an inverse EKE cascade through nonlinear eddy interactions. This is examined here by generalizing the techniques explored in SW05 to retain directional information. Below we derive the balance equations for the EKE power spectral density, $\bar{E}\left(k_{x}, k_{y}, t\right)$, as a function of zonal and meridional wavenumber, $k_{x}$ and $k_{y}$. While most of the terms are not directly measurable, the rate of forcing of $\bar{E}\left(k_{x}, k_{y}, t\right)$ by 
horizontal advection (or "eddy-eddy interactions"), $\bar{T}\left(k_{x}, k_{y}, t\right)$, is measurable, and forms our main diagnostic.

\section{a. Spectral evolution equations}

Consider the horizontal momentum equations on a Cartesian section of the rotating earth:

$$
\begin{aligned}
& \frac{\partial u}{\partial t}+u_{j} \frac{\partial u}{\partial x_{j}}-f v=-\frac{1}{\rho} \frac{\partial p}{\partial x}+\operatorname{Fr}^{x}, \\
& \frac{\partial v}{\partial t}+u_{j} \frac{\partial v}{\partial x_{j}}+f u=-\frac{1}{\rho} \frac{\partial p}{\partial y}+\operatorname{Fr}^{y},
\end{aligned}
$$

where index $i=1,2$ indicates the $x, y$ directions, respectively, and $\mathrm{Fr}^{x}$ and $\mathrm{Fr}^{y}$ are the frictional terms, including any vertical advection of momentum. Taking the discrete Fourier transform (DFT) of Eqs. (8) and (9), multiplying by the respective complex conjugates, $\hat{u}^{*}$ and $\hat{v}^{*}$, where the star indicates complex conjugate and caret indicates DFT, and finally summing we obtain

$$
\frac{\partial E\left(k_{x}, k_{y}, t\right)}{\partial t}=T\left(k_{x}, k_{y}, t\right)+P\left(k_{x}, k_{y}, t\right)-D\left(k_{x}, k_{y}, t\right),
$$

where the power spectral density is

$$
E\left(k_{x}, k_{y}, t\right) \equiv \frac{1}{2}\left(\hat{u} \hat{u}^{*}+\hat{v} \hat{v}^{*}\right) / \Delta k^{2},
$$

with $\Delta k=1 / N \Delta x \mathrm{cpkm}$, where $\Delta x$ is the grid spacing and $N=32$ is the number of grid points in each direction. The horizontal wavenumber vector is $\left(k_{x}, k_{y}\right)=$ $2 \pi\left(m / L_{x}, n / L_{y}\right)$, where $m, n \in[-32,32]$. The horizontal advection terms give rise to the spectral energy transfer:

$$
T\left(k_{x}, k_{y}, t\right) \equiv-\Re\left[\hat{u}^{*} u_{j} \widehat{\frac{\partial u}{\partial x_{j}}}+\hat{v}^{*} u_{j} \frac{\partial v}{\partial x_{j}}\right] / \Delta k^{2} .
$$

In Eq. (12), the caret again indicates DFT. Physically, $T\left(k_{x}, k_{y}, t\right)$ represents the redistribution of EKE between different spatial modes due to eddy-eddy interactions; it can be estimated from the altimetrically derived SSH data by assuming geostrophy. In Eq. (10), $P\left(k_{x}, k_{y}, t\right)$ includes the rate of conversion of potential to kinetic energy and the dissipation term $D\left(k_{x}, k_{y}, t\right)$ arises from the frictional terms, with unknown functional form.

Taking the bimonthly average of Eq. (10), we find $\partial \bar{E}\left(k_{x}, k_{y}, t\right) / \partial t$ based on the SSH-derived $E\left(k_{x}, k_{y}, t\right)$ time series is about an order of magnitude smaller than
$\bar{T}\left(k_{x}, k_{y}, t\right)$ (to be shown in Fig. 10). In this case, the following approximate balance of terms becomes valid:

$$
\bar{T}\left(k_{x}, k_{y}, t\right) \approx-\bar{P}\left(k_{x}, k_{y}, t\right)+\bar{D}\left(k_{x}, k_{y}, t\right),
$$

where the overbar denotes the bimonthly temporal average. Equation (13) provides the key for interpreting our results. Presumably the dissipation term $\bar{D}\left(k_{x}, k_{y}, t\right)$ dominates only at high wavenumber $K^{2}=k_{x}^{2}+k_{y}^{2}$. So at low $K$, the spectral transfer of EKE into wavenumber modes $\left(k_{x}, k_{y}\right)$ is mostly balanced by the forcing term $\bar{P}\left(k_{x}, k_{y}, t\right)$. Indeed we support this interpretation below by comparing $\bar{T}\left(k_{x}, k_{y}, t\right)$ with the baroclinic instability calculations of section 3 .

\section{b. Results}

Figure 10 shows the bimonthly maps of the spectral transfer term $\bar{T}\left(k_{x}, k_{y}, t\right)$ as a function of zonal and meridional wavenumbers. The white circles in each map delineate the boundaries within which the perturbations are subject to baroclinic instability of the vertically sheared STCC-SEC system. Here, the size of the unstable window modulates due to the seasonal changes in the zonal mean velocity and density in the surface STCC layer (i.e., $U_{1}$ and $\rho_{1}$ ). In AugustSeptember when the regional condition for baroclinic instability is most favorable, Fig. 10 reveals that $\bar{T}\left(k_{x}\right.$, $\left.k_{y}, t\right)$ inside the unstable window is largely negative, especially in areas where $k_{y}$ is small. The $\bar{T}\left(k_{x}, k_{y}, t\right)$ pattern, in fact, mirrors quite well the growth rate pattern shown in Fig. 9a, suggesting that baroclinic instability is the energy source [i.e., the $\bar{P}$ term in Eq. (13)] that elevates the regional EKE level, as well as contributes to the nonlinear eddy interactions. It is interesting to note that throughout the seasons, $\bar{T}\left(k_{x}, k_{y}, t\right)$ remains mostly negative inside the unstable window and this is especially true in areas where $k_{y}$ is small. This result implies that while its intensity modulates with seasons, baroclinic instability of the STCC-SEC system provides a continual energy input for the spectral transfer term.

It is also clear from Fig. 10 that most of the kinetic energy is transferred to larger scales, and especially larger zonal scales, consistent with Scott and Wang (2005). Note the strongly positive $\bar{T}\left(k_{x}, k_{y}, t\right)$ values seen in areas with horizontal wavelengths $2 \pi / K>300$ $\mathrm{km}$ and that they tend to have a preference for small $k_{x}$ values. This suggests an anisotropic, inverse cascade transferring EKE from meridionally elongated modes $\left(k_{x}>k_{y}\right)$ to zonally elongated modes $\left(k_{y}>k_{x}\right)$. To confirm this we integrated $\bar{T}\left(k_{x}, k_{y}, t\right)$ over the regions $k_{y}>k_{x}$ to form

$$
T_{z}=\sum_{k_{y}>k_{x}} \bar{T}\left(k_{x}, k_{y}, t\right) \Delta k^{2},
$$



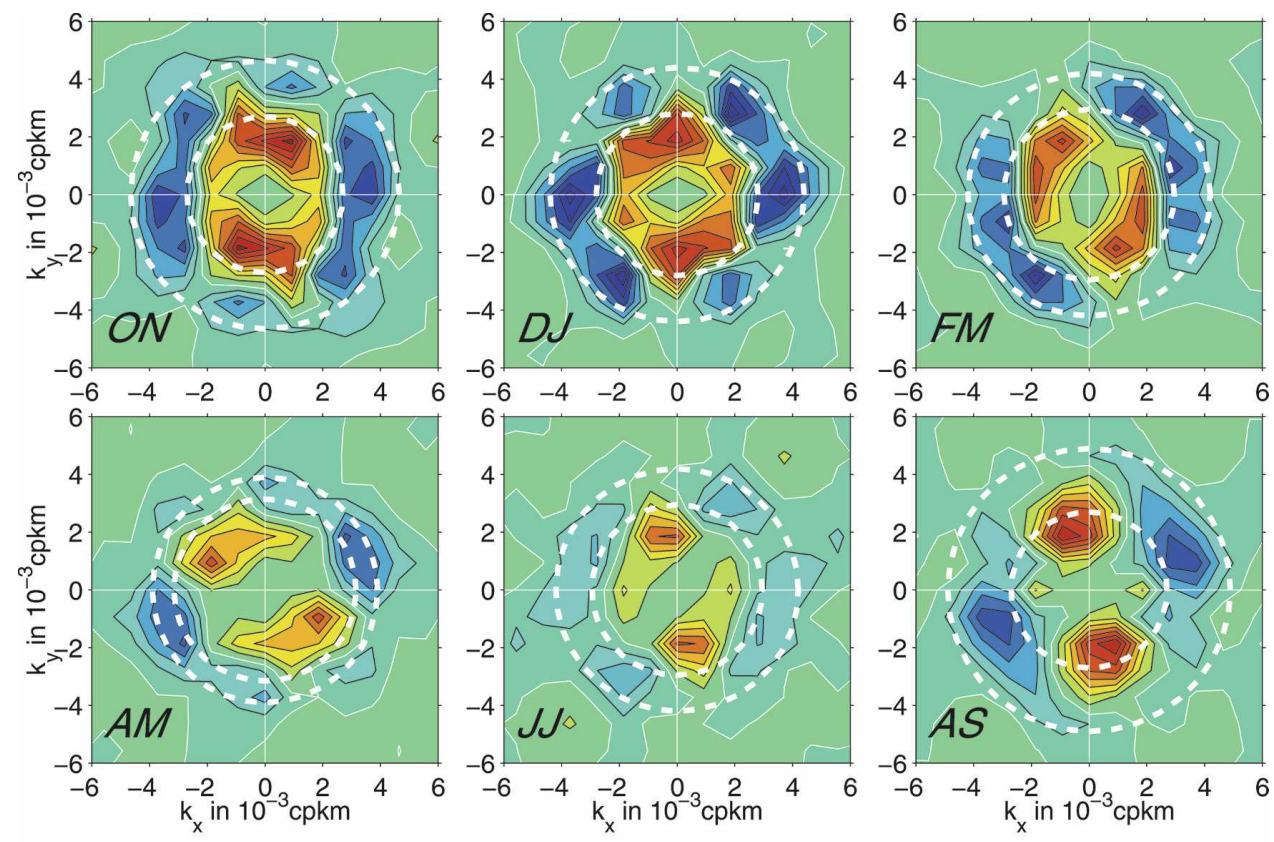

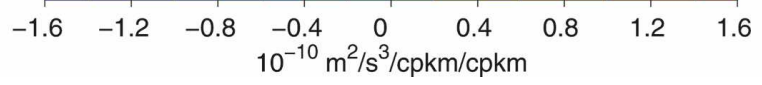

FIG. 10. Bimonthly spectral energy transfer $\bar{T}\left(k_{x}, k_{y}, t\right)$ in the South Pacific STCC band of $20^{\circ}-30^{\circ} \mathrm{S}$, $170^{\circ} \mathrm{E}-130^{\circ} \mathrm{W}$; see Eq. (12) for the definition. White dashed circles denote the boundaries within which perturbations are baroclinically unstable, given the bimonthly mean flow structure of the observed STCC-SEC. The letters ON-AS are defined as in Fig. 4.

where $\bar{T}\left(k_{x}, k_{y}, t\right)$ are the bimonthly averages shown in Fig. 10. Physically, $T_{z}$ represents transfer of EKE from meridionally elongated modes into zonally elongated modes by nonlinear eddy interactions. In Fig. 11 we see

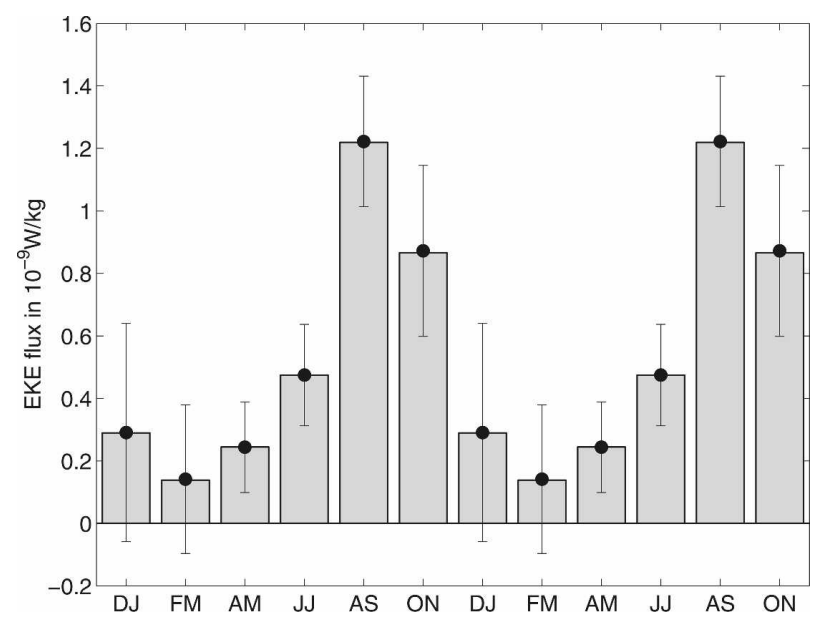

FIG. 11. Rate of EKE transfer from meridionally elongated to zonally elongated modes, $T_{z}$ [see Eq. (14)]. Error bars represent the std dev divided by $\sqrt{N-1}$, where $N$ is the number of available $T_{z}$ estimates in the bimonthly bins. that indeed this forcing is always positive. This nonlinear transfer of EKE between meridionally and zonally elongated modes appears to have a regular seasonal cycle, peaking between August and November. These are the months with strongest baroclinic instability (largest sizes of the instability circles in Fig. 10), so we expect the greatest generation of EKE. Because the baroclinic instability preferentially generates meridional elongated anomalies, it is during August through November that the nonlinear transfer via triad eddy interaction works most effectively to redistribute EKE into zonally elongated anomalies. For instance, the large $T_{z}$ value in October-November of Fig. 11 explains why in Fig. 5 the $\left(\mathrm{EKE}_{z}-\mathrm{EKE}_{m}\right) / \mathrm{EKE}$ value changes rapidly from negative in October-November to positive in December-January.

The anisotropic cascade in Figs. 10 and 11 explains why we found in Figs. 4 and 5 that $\mathrm{EKE}_{z}>\mathrm{EKE}_{m}$ for all months except October-November, despite the fact that the most baroclinically unstable modes have $k_{x}>$ $k_{y}$. With the exception for October-November, we can infer that the eddy-eddy interaction forcing is too strong to achieve a more meridionally aligned flow. As discussed in the introduction, we expect such an aniso- 
tropic inverse cascade for the barotropic mode of geostrophic turbulence (e.g., Rhines 1977; Vallis and Maltrud 1993; Galperin et al. 2004, 2006). It would appear that the $\beta$ effect is capable of redirecting the inverse baroclinic kinetic energy cascade (SA07) as well.

As discussed in SW05, the sink of this energy is not clear. It seems unlikely that dissipation $\bar{D}\left(k_{x}, k_{y}, t\right)$ dominates at these large length scales, though admittedly vertical advection of momentum was included in this term and remains largely unknown. SA07 find that barotropization can arrest the surface layer cascade in QG dynamics. If applicable here, then Eq. (13) suggests that the positive $\bar{T}\left(k_{x}, k_{y}, t\right)$ term has to be balanced by $\bar{P}\left(k_{x}, k_{y}, t\right)$, the latter being dominated by the vertical transfer of energy. With only observed SSH information, it is difficult to verify this speculation in the present study.

\section{Summary and discussions}

Multiple satellite altimeter missions over the past 14 yr provide us with an unprecedented SSH dataset to investigate the surface ocean variability on various temporal and spatial scales. In this study, we focused on the Subtropical Countercurrent (STCC) in the South Pacific where the regional mesoscale eddy field is dominated by large-amplitude seasonal modulations. Based on the stability analysis of the time-varying mean flow system, as well as the inferred triad eddy interactions, we can separate the observed eddy evolution into three dynamic phases.

\section{a. Growing phase (August-October)}

Starting in August when the vertical shear between the eastward-flowing STCC and the westward-flowing SEC reaches the seasonal maximum, the flow system baroclinic instability peaks. Because STCC and SEC are relatively weak, interior-ocean currents, the growth rate of the instability is modest and it takes several months for initial perturbations to attain finite amplitudes. This modest growth leads to a delayed, regional EKE maximum in December. The baroclinic instability preferentially generates meridionally elongated perturbations with greater $v$ than $u$ variance. So in OctoberNovember, before the inverse cascade has fully redistributed the EKE, the flow is actually more meridionally elongated; see maximum in $\bar{E}\left(k_{x}, k_{y}, t\right)$ at large $k_{x}$ and small $k_{y}$ in Fig. 4.

\section{b. Maturing phase (November-January)}

As the instability-induced perturbations attain finite amplitude in this stage, an active inverse cascade takes place. As the EKE level equilibrates during this phase, the energy input supplied by baroclinic instability is balanced by the spectral energy transfer term, $\bar{T}\left(k_{x}, k_{y}\right.$, $t)$. Because of the cumulative energy transfers from meridional to zonal scales over the growing phase, the peak $\bar{E}\left(k_{x}, k_{y}, t\right)$ value shifts to small $k_{x}$, and $k_{y}>k_{x}$ during this maturing phase of eddy evolution.

\section{c. Decaying phase (March-June)}

Energy input from baroclinic instability during this phase is weak due to weakened vertical shear between the STCC and SEC. Without the meridionally elongated anomalies supplied by the instability, the inverse cascade into the zonal anomalies wins out during this more "freely evolving" phase. As in the other two phases, triad eddy interaction works to transfer energy to larger scales where the nonlinear interaction term now acts as an energy sink.

Several findings from this study are worth emphasizing. First, our analysis confirms that the seasonally modulating baroclinic instability of the STCC-SEC system is the energy source for the eddy generation and their subsequent interactions. This latter point was speculated by Scott and Wang (2005) and our stability analysis helps to verify it. Second, due to the instability nature of the zonal mean flow, baroclinic instability favors the formation of meridionally elongated anomalous flows. Third, the nonlinear eddy-eddy interaction results in an inverse energy cascade with a preference of transferring kinetic energy from the anomalous meridional flows to the zonal ones. This anisotropic energy cascade into anomalies with $k_{y}>k_{x}$ is consistent with Vallis and Maltrud (1993) and is likely a result of the $\beta$ effect. Although this inverse cascade is analogous to the classic barotropic inverse cascade (Rhines 1975), we speculate that it represents the baroclinic inverse kinetic energy cascade (SW05; SA07). Notice that in the STCC-SEC system (where $U_{1}>0$ and $U_{2}<0$ ), Eq. (7) indicates that the planetary $\beta$ is enhanced by the vertically sheared STCC-SEC mean flow in the surface layer and it is weakened in the subsurface layer.

Although our analysis of eddy diagnostic supports the upscale energy cascade, the net outcome for the formation of surface zonal flows is rather limited. As we presented in Fig. 5, the zonally elongated EKE values in all seasons do not exceed the meridionally elongated EKE values by more than 5\%. This is in contrast to the findings by Maximenko et al. (2005) who showed zonal jetlike structures in the subtropical gyres of the World Ocean, which we believe arise mostly from a very different mechanism. In their study, the zonal jetlike structures emerge in velocity maps with a timeaveraging window of 18 weeks. As mesoscale eddies 
(a) No averaging

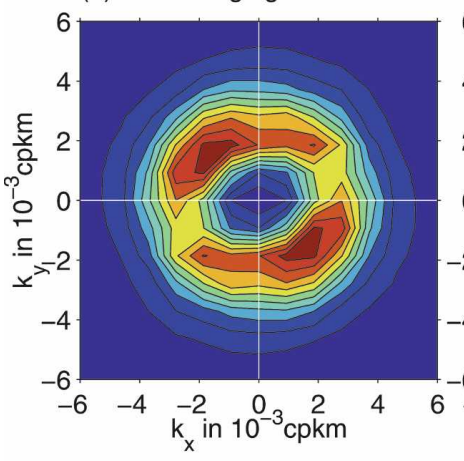

(b) 9-weeks averaging

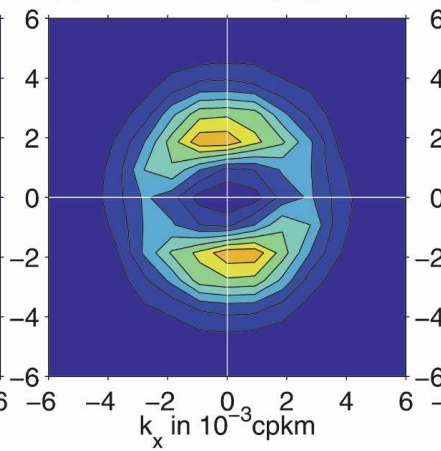

(c) 18-weeks averaging

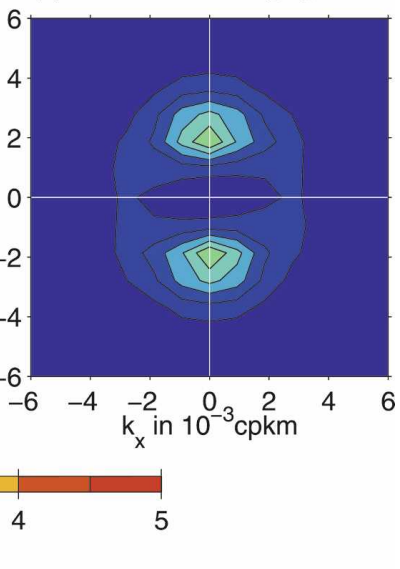

FIG. 12. EKE power spectral density distributions in the $10^{\circ} \times 10^{\circ}$ box centered on $25^{\circ} \mathrm{S}, 180^{\circ}$ in the STCC band: (a) based on the original weekly SSH data; (b) based on the SSH data averaged over a 9-week window; (c) based on the SSH data averaged over an 18-week window. The integrated EKE levels in the box from (a), (b), and (c) are 129, 62, and $34 \mathrm{~cm}^{2} \mathrm{~s}^{-2}$, respectively.

tend to move due west with speed close to baroclinic Rossby waves (e.g., Chelton et al. 2007; Fu and Chelton 2001), caution is required when interpreting the Eulerian-averaged, westward-translating mesoscale anomaly signals (see also Huang et al. 2007). To quantify the effect of time averaging, we plot in Fig. 12 the two-dimensional EKE power spectral density distributions when the weekly SSH data in the South Pacific STCC region was (a) treated independently, (b) averaged with a 9-week window, and (c) averaged with a 18-week window. The averaging effect is dramatic: while (a) based on the original weekly SSH data shows nearly isotropic EKE signals, (b) and (c) indicate a progressive domination by zonally aligned signals. The mechanism of these time-averaged, jetlike structures appears to be largely kinematic ${ }^{2}$ and is very different from the dynamic interactions predicted by turbulence theory and diagnosed in Figs. 10 and 11. Note further that these kinematic jets are buried in a much stronger EKE field: the kinetic energy levels in Figs. 12b and 12c are, respectively, only $48 \%$ and $27 \%$ of the value in Fig. 12a. In idealized, $\beta$-plane, quasigeostrophic turbulence modeling studies, no time averaging is required to reveal the zonal jets of dynamical origin; the dominance of the zonal velocity over the meridional velocity is readily apparent in individual snapshots (cf. Fig. 6 of

\footnotetext{
${ }^{2}$ By "kinematic," we mean arising from the statistics of the velocity field, and without invoking any nonlinear interactions in the equations of motion. Of course, the dynamics that produced the velocity field statistics, and the westward-propagating eddies in particular, can be nonlinear.
}

Rhines 1975; Fig. 7 of Panetta 1993; and Fig. 1a of Okuno and Masuda 2003).

Despite the fact that the eddy-eddy interaction works to form zonally elongated anomalies, satellite altimeter data show that only limited zonally aligned anomalies emerge. We see three possible explanations for this "paradox." First, the upper ocean might be too diffusive; although we do not have the needed data to refute this scenario, we find this unlikely to be the leading cause for the decay of the large-scale EKE signals (recall Fig. 3). The second possibility is that before the zonal mean flow patterns can establish themselves during the decaying period of eddies, the flow field is taken over by the newly erupted baroclinic instability, which as we noted through this study, favors meridionally elongated anomalies. The third plausible scenario is that the EKE is being transferred downward (barotropized), while it is being cascaded horizontally during the eddy decaying period. With the SSH information alone, it is difficult to ascertain this process, although its occurrence following baroclinic instability of zonal flow systems has been detected in many idealized primitive equation (Halliwell et al. 1994) and geostrophic turbulence modeling studies (e.g., Hua and Haidvogel 1986; Arbic and Flierl 2004; SA07). It would be interesting to explore this scenario in a model with a seasonally modulating zonal mean flow system in light of our analysis based on the satellite altimeter data.

Acknowledgments. This study benefited from discussions with Brian Arbic, Dudley Chelton, and Lee Fu. Detailed comments made by the anonymous reviewers helped improve an early version of the manuscript. The 
merged satellite altimeter data are provided by the CLS Space Oceanography Division as part of the Environment and Climate EU ENACT project. The hydrographic data are provided by NOAA's National Ocean Data Center. BQ and SC were supported by NASA through Contract 1207881. RBS was supported by NSF Grant OCE-0526412.

\section{REFERENCES}

Antonov, J. I., R. A. Locarnini, T. P. Boyer, A. V. Mishonov, and H. E. Garcia, 2006: Salinity. Vol. 2, World Ocean Atlas 2005, NOAA Atlas NESDIS 62, $182 \mathrm{pp}$

Arbic, B. K., and G. R. Flierl, 2004: Baroclinically unstable geostrophic turbulence in the limits of strong and weak bottom Ekman friction: Application to midocean eddies. J. Phys. Oceanogr., 34, 2257-2273.

Bowen, M. M., J. L. Wilkin, and W. J. Emery, 2005: Variability and forcing of the East Australian Current. J. Geophys. Res., 110, C03019, doi:10.1029/2004JC002533.

Charney, J., 1971: Geostrophic turbulence. J. Atmos. Sci., 28, 1087-1095.

Chelton, D. B., M. G. Schlax, R. M. Samelson, and R. A. de Szoeke, 2007: Global observations of large oceanic eddies. Geophys. Res. Lett., 34, L15606, doi:10.1029/2007GL030812.

Danilov, S. D., and D. Gurarie, 2000: Quasi-two-dimensional turbulence. Phys. Uspekhi, 43, 863-900.

Ducet, N., P.-Y. Le Traon, and G. Reverdin, 2000: Global highresolution mapping of ocean circulation from TOPEX/ Poseidon and ERS-1 and -2. J. Geophys. Res., 105, $19477-$ 19498.

Frisch, U., 1995: Turbulence: The Legacy of A. N. Kolmogorov. Cambridge University Press, 296 pp.

Fu, L.-L., and G. R. Flierl, 1980: Nonlinear energy and enstrophy transfers in a realistically stratified ocean. Dyn. Atmos. Oceans, 4, 219-246.

- and D. B. Chelton, 2001: Large-scale ocean circulation. Satellite Altimetry and Earth Sciences, L.-L. Fu and A. Cazenava, Eds., Academic Press, 133-169.

Galperin, B., H. Nakano, H.-P. Huang, and S. Sukoriansky, 2004: The ubiquitous zonal jets in the atmospheres of giant planets and earth's oceans. Geophys. Res. Lett., 31, L13303, doi:10.1029/2004GL019691.

, S. Sukoriansky, N. Dikovskaya, P. L. Read, Y. H. Yamazaki, and R. Wordsworth, 2006: Anisotropic turbulence and zonal jets in rotating flows with a beta-effect. Nonlinear Processes Geophys., 13, 83-98.

Halliwell, G. R., Jr., G. Peng, and D. B. Olson, 1994: Stability of the Sargasso Sea subtropical frontal zone. J. Phys. Oceanogr., 24, 1166-1183.

Hua, B. L., and D. B. Haidvogel, 1986: Numerical simulations of the vertical structure of quasi-geostrophic turbulence. J. Atmos. Sci., 43, 2923-2936.

Huang, H.-P., A. Kaplan, E. N. Curchister, and N. A. Maximenko, 2007: The degree of anisotropy for mid-ocean currents from satellite observations and an eddy-permitting model simulation. J. Geophys. Res., 112, C09005, doi:10.1029/ 2007JC004105.

Huang, R. X., and B. Qiu, 1998: The structure of the wind-driven circulation in the subtropical South Pacific. J. Phys. Oceanogr., 28, 1173-1186.
Kobashi, F., and H. Kawamura, 2002: Seasonal variation and instability nature of the North Pacific Subtropical Countercurrent and the Hawaiian Lee Countercurrent. J. Geophys. Res., 107, 3185, doi:10.1029/2001JC001225.

Lapeyre, G., and P. Klein, 2006: Dynamics of the upper oceanic layers in terms of surface quasigeostrophy theory. J. Phys. Oceanogr., 36, 165-176.

Larichev, V. D., and I. M. Held, 1995: Eddy amplitudes and fluxes in a homogeneous model of fully developed baroclinic instability. J. Phys. Oceanogr., 25, 2285-2297.

Le Traon, P.-Y., and R. Morrow, 2001: Ocean currents and eddies. Satellite Altimetry and Earth Sciences, L.-L. Fu and A. Cazenava, Eds., Academic Press, 171-215.

—_, F. Nadal, and N. Ducet, 1998: An improved mapping method of multi-satellite altimeter data. J. Atmos. Oceanic Technol., 25, 522-534.

Locarnini, R. A., A. V. Mishonov, J. I. Antonov, T. P. Boyer, and H. E. Garcia, 2006: Temperature. Vol. 1, World Ocean Atlas 2005, NOAA Atlas NESDIS 61, 182 pp.

Maximenko, N. A., B. Bang, and H. Sasaki, 2005: Observational evidence of alternating zonal jets in the World Ocean. Geophys. Res. Lett., 32, L12607, doi:10.1029/2005GL022728.

Morrow, R., and P.-Y. Le Traon, 2006: Fifteen years of satellite altimetry and mesoscale eddy dynamics. Proc. 15 Years of Progress in Radar Altimetry Symp., Venice, Italy, ESA. [Available online at http://earth.esa.int/workshops/venice06/ participants/889/paper_889_morrow-v2.pdf.]

Okuno, A., and A. Masuda, 2003: Effect of horizontal divergence on the geostrophic turbulence on a beta-plane: Suppression of the Rhines effect. Phys. Fluids, 15, 56-65.

Panetta, R. L., 1993: Zonal jets in wide baroclinically unstable regions: Persistence and scale selection. J. Atmos. Sci., 50, 2073-2106.

Pedlosky, J., 1987: Geophysical Fluid Dynamics. Springer-Verlag, 710 pp.

Qiu, B., 1999: Seasonal eddy field modulation of the North Pacific Subtropical Countercurrent: TOPEX/Poseidon observations and theory. J. Phys. Oceanogr., 29, 2471-2486.

- and S. Chen, 2004: Seasonal modulation in the eddy field of the South Pacific Ocean. J. Phys. Oceanogr., 34, 1515-1527.

Rhines, P. B., 1975: Waves and turbulence on a beta-plane. $J$. Fluid Mech., 69, 417-443.

—, 1977: The dynamics of unsteady currents. The Sea, E. D. Goldberg et al., Eds., Vol. 6, Marine Modeling, John Wiley and Sons, 189-318.

Robinson, A. R., 1983: Eddies in Marine Science. Springer-Verlag, $609 \mathrm{pp}$.

Roemmich, D., and B. Cornuelle, 1990: Observing the fluctuations of gyre-scale ocean circulation: A study of the subtropical South Pacific. J. Phys. Oceanogr., 20, 1919-1934.

— and J. Gilson, 2001: Eddy transport of heat and thermocline waters in the North Pacific: A key to interannual/decadal climate variability? J. Phys. Oceanogr., 31, 675-687.

Rotschi, H., 1973: Hydrography at $170^{\circ} \mathrm{E}$ in the South Pacific. Oceanography of the South Pacific 1972, New Zealand National Commission for UNESCO, 113-128.

Salmon, R., 1980: Baroclinic instability and geostrophic turbulence. Geophys. Astrophys. Fluid Dyn., 15, 167-211. , 1998: Lectures on Geophysical Fluid Dynamics. Oxford University Press, $378 \mathrm{pp}$.

Scott, R. B., and F. Wang, 2005: Direct evidence of an oceanic inverse kinetic energy cascade from satellite altimetry. $J$. Phys. Oceanogr., 35, 1650-1666. 
and B. K. Arbic, 2007: Spectral energy fluxes in geostrophic turbulence: Implications for ocean energetics. J. Phys. Oceanogr., 37, 673-688.

Smith, K. S., and G. K. Vallis, 2001: The scales and equilibration of midocean eddies: Freely evolving flow. J. Phys. Oceanogr., 31, 554-571.

, and — 2002: The scales and equilibration of midocean eddies: Forced dissipative flow. J. Phys. Oceanogr., 32, 16991720.
Vallis, G. K., 2006: Atmospheric and Oceanic Fluid Dynamics: Fundamentals and Large-Scale Circulation. Cambridge University Press, 744 pp.

— , and M. E. Maltrud, 1993: Generation of mean flows and jets on a beta plane and over topography. J. Phys. Oceanogr., 23, 1346-1362.

Wunsch, C., 1997: The vertical partition of oceanic horizontal kinetic energy and the spectrum of global variability. J. Phys. Oceanogr., 27, 1770-1794. 